\title{
Considérations actuelles sur les effets physiopathologiques des infra-sons
}

\author{
P. BORREDON $(*)$, P. QUANDIEU $(*)$ \\ (Manuscrit reçu le 3 décembre 1976)
}

\begin{abstract}
RÉSUMÉ
Les effets physiopathologiques des infra-sons sont envisagés dans le cadre des effets énergétiques des vibrations mécaniques aériennes. Les données expérimentales actuellement disponibles et concernant l'homme et l'animal sont présentées et discutées. Des normes de tolérance qui ont pu être dégagées à l'issue d'un premier colloque international sont rapportées.
\end{abstract}

\begin{abstract}
The physiopathologic effects of infra-sounds are considered from the point of view of the energetic effects of air transmitted mechanical vibrations. The experimental data presently available about man and animal are presented and discussed. The tolerance standards which were drawn from a first international symposium are reported.
\end{abstract}

\section{I. - INTRODUCTION}

Sous le terme d'infra-sons sont considérées ici les vibrations mécaniques aériennes de fréquences inférieures à $20 \mathrm{~Hz}$. Le système matériel en vibration est la molécule de gaz qui oscille de part et d'autre de sa position d'équilibre au sein d'un milieu élastique, l'air. Chaque molécule en vibration transmet son énergie

(*) École d'Application du Service de Santé pour l'Armée de l'Air et Centre de Recherches de Médecine Aéronautique, 26, boulevard Victor, 75753 Paris Cedex 15. 
vibratoire aux molécules voisines, et le phénomène se traduit globalement, en fonction du temps $t$, par une variation de pression, $\Delta P(t)$, autour de la pression ambiante $i$.e. le plus souvent de la pression atmosphérique $P_{B Z}$. A chaque instant, la pression en un point est de la forme

$$
P(t)=P_{B Z}+\Delta P(t) .
$$

L'ébranlement de pression se propage dans l'air avec une certaine vitesse, la célérité $C$. La célérité ne dépend que du milieu considéré; elle est indépendante de la fréquence. La vitesse de propagation de l'ébranlement de pression infrasonore est donc identique à la célérité du son; dans l'air à $20^{\circ} \mathrm{C}$, elle est voisine de $340 \mathrm{~m} / \mathrm{s}$.

L'organisme humain dispose d'un capteur différentiel de pression aérienne : l'oreille. Ce capteur est sensible au terme $\Delta P(t)$ de l'équation (1). La bande passante de ce capteur est le critère qui a été choisi pour définir les vibrations sonores de fréquences comprises entre 20 et $20000 \mathrm{~Hz}$, les ultra-sons de fréquence supérieure à $20 \mathrm{kHz}$ et les infra-sons de fréquence inférieure à $20 \mathrm{~Hz}$ [25].

Les vibrations mécaniques aériennes peuvent être entretenues ou non entretenues. Les vibrations non entretenues ou transitoires correspondent, en général, à un mouvement de grande amplitude et le phénomène n'est constitué que par une onde de variation de pression importante, rapidement amortie. Des exemples en sont donnés par le bang ou les bruits d'arme. Dans le cas d'un bang où l'onde " en $N$ » a une durée de l'ordre de $100 \mathrm{~ms}$ (avion de chasse) à $300 \mathrm{~ms}$ (transport supersonique), les premiers termes du spectre de Fourier sont très importants et les composantes infra-sonores du spectre comportent la majeure partie de l'énergie [28]. Or, si au cours de leur propagation en milieu aérien les ultra-sons sont rapidement absorbés, il n'en est pas de même des sons graves et a fortiori des infra-sons. A grande distance, finalement, ne subsistent que les composantes infra-sonores du spectre.

En ce qui concerne les vibrations entretenues, on sait que l'évolution de la technologie, dans le domaine des réacteurs notamment, fait que la répartition spectrale des énergies sonores de ces sources de bruit se déplace de plus en plus vers les fréquences graves : « plus un réacteur a un gros diamètre, plus il a la voix grave ". Les espaces clos, fixes ou mobiles, qui sont ventilés par de l'air pulsé à fréquence basse sont de plus en plus nombreux. Les hauts-fourneaux, les cowpers, les gros moteurs diesels représentent autant de sources industrielles d'infra-sons.

Finalement, il existe un très grand nombre de sources naturelles (vents, tonnerre, tremblements de terre, etc.) ou techniques d'infra-sons $[1,28]$ et ces quelques considérations préliminaires montrent que s'interroger sur les problèmes posés par les effets physiopathologiques des infra-sons n'est pas se complaire dans le domaine de la spéculation. Mais de là à vouloir expliquer les mystères du triangle des Bermudes $[18,20]$ ou certains accidents inexpliqués de la circulation [7] par l'invocation du phénomène infra-sonore, il $\mathrm{y}$ a un pas qu'il faut, peut-être, éviter de franchir, compte tenu des premiers résultats 
expérimentaux actuellement en notre possession. Ces éléments sont encore peu nombreux. Ils permettent, cependant, de réduire le mythe infra-sonore à des proportions plus modestes et d'envisager, avec une certaine sérénité, les problèmes posés par la pollution infra-sonore $[1,30]$.

\section{II. - LE CADRE DES EFFETS PHYSIOPATHOLOGIQUES DES VIBRATIONS ACOUSTIQUES}

Les effets physiopathologiques des vibrations acoustiques peuvent, schématiquement, être envisagés sous trois aspects [5] :

- un aspect énergétique quand l'intensité du stimulus sonore est telle que le capteur sensoriel risque d'être lésé. C'est tout le chapitre de la surdité professionnelle;

- un point de vue informationnel quand le capteur sensoriel est excité dans une bande passante et dans une dynamique qui lui sont adaptées. Ces effets sont rencontrés quand l'intensité du stimulus sonore n'est pas suffisante pour être dangereuse pour la cochlée. Le capteur sensoriel transmet intégralement le message vers les centres supérieurs d'intégration. Cet afflux de sollicitations sensorielles entraîne un certain nombre d'effets correspondant sensiblement à la notion de gêne;

- l'aspect global, enfin, des effets généraux sur l'organisme d'un agent physique de l'environnement dont les caractères en font, éventuellement, un agent agressif.

Dans le cas des vibrations sonores, ces effets généraux sont des effets mixtes, à la fois d'ordre énergétique et d'ordre informationnel. Une vibration aérienne audible dont les caractères permettent de prévoir qu'elle aura des effets essentiellement informationnels pourra, indirectement, par l'intermédiaire de phénomènes psychosomatiques, solliciter la mise en jeu et entraîner un dérèglement des grandes fonctions neuro-végétatives. Le schéma de GRANDJEAN (fig. 1) illustre parfaitement cette conception [14]. Dans le cas des infra-sons dont le domaine est, par définition, en dehors de la bande passante de l'oreille humaine, cette éventualité ne paraît pas devoir être envisagée. Par contre, de façon directe, la sollicitation du tympan par un stimulus vibratoire aérien intense et de basse fréquence peut mettre en jeu l'innervation sympathique de la caisse. Cette éventualité doit être particulièrement prise en compte quand il s'agit d'une vibration sonore très grave, voire infra-sonore, qui mobilise le tympan sans donner lieu à une réponse auditive spécifique mais plutôt à une impression tactile, la partie sensorielle du capteur n'étant ni excitée, ni lésée. Enfin, l'hypothèse peut être émise que des fluctuations infra-sonores de grande amplitude, entraînant des variations importantes des pressions au sein de l'endolymphe cochléaire, puissent déterminer une excitation vestibulaire inopportune [27].

De manière encore plus directe, le phénomène vibratoire infra-sonore propagé peut solliciter directement tout ou partie de l'organisme. Ces parties peuvent être, par exemple, les parois des cavités abdominales ou thoraciques ou 
leur contenu aérien ou les organes suspendus par leur pédicule à l'intérieur de ces cavités. On sait que, chezl'homme, les fréquences de résonance de ces éléments se trouvent être dans la gamme infra-sonore [12].

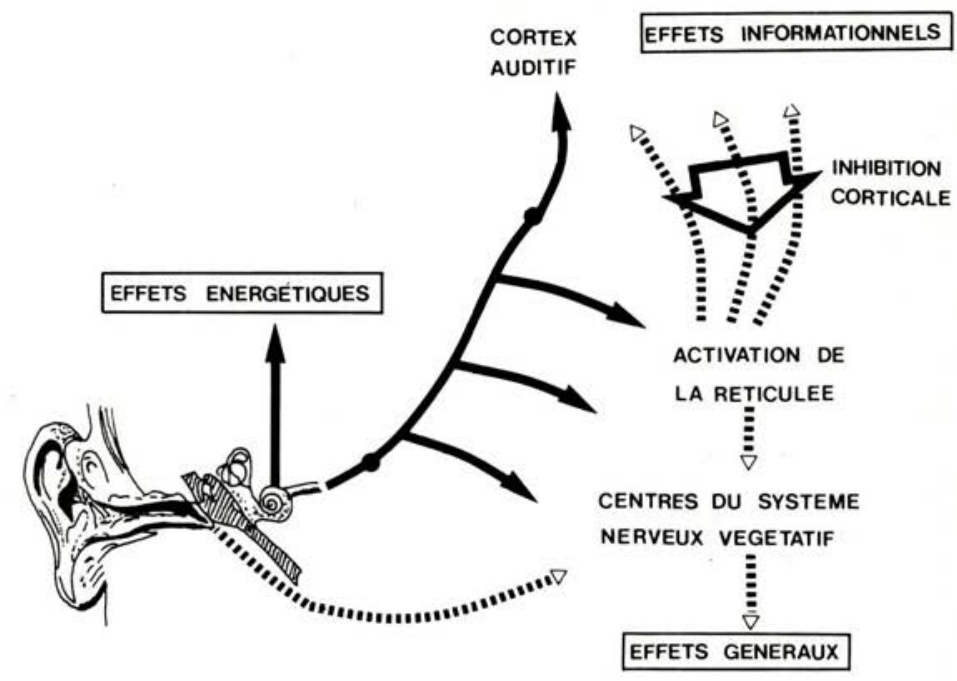

FIG. 1. - Les trois aspects des effets du bruit sur l'organisme humain (schéma modifié d'après Grandjean [14]).

Finalement, dans le domaine infra-sonore, il n'est pas évident que le capteur sensoriel qu'est l'oreille, au sens large du terme, soit le système qui, vis-à-vis de l'agression, ait le seuil de réponse ou le seuil lésionnel le plus bas. Il semble donc que, dans ce domaine, les effets à envisager soient des effets généraux et que ces effets, si effet il y a, soient essentiellement d'ordre énergétique.

\section{III. - DONNÉES EXPÉRIMENTALES}

Comme le fait remarquer Pimonow dans le très bel ouvrage qu'il vient de consacrer aux infra-sons [28], les données expérimentales actuellement disponibles sont peu nombreuses, comparées à celles, innombrables, publiées dans le domaine sonore.

\section{$1^{\circ}$ Les Dispositifs EXPÉRIMENTAuX}

Une remarque doit être faite d'emblée à propos des dispositifs expérimentaux qui ont été utilisés pour les expériences de biologie. Il s'agit pratiquement toujours de chambres closes dans lesquelles un piston crée des variations périodiques de pression. Un exemple en est donné par le dispositif expérimental que nous avons nous-mêmes utilisé. Il s'agit essentiellement d'un 
caisson rigide (fig. 2) dont l'une des parois verticales comporte un panneau mobile entraîné par un piston hydraulique qui lui communique un mouvement sinusoïdal. Le volume de l'enceinte est d'environ $6,3 \mathrm{~m}^{3}$ (base hexagonale de $1,10 \mathrm{~m}$ de côté et de $2 \mathrm{~m}$ de hauteur). Au-dessus du panneau mobile, un hautparleur vient s'encastrer dans une seconde ouverture de la paroi postérieure du caisson. Le piston hydraulique crée à l'intérieur de l'enceinte une variation de pression sinusoïdale de fréquence infra-sonore. L'étude de la répartition

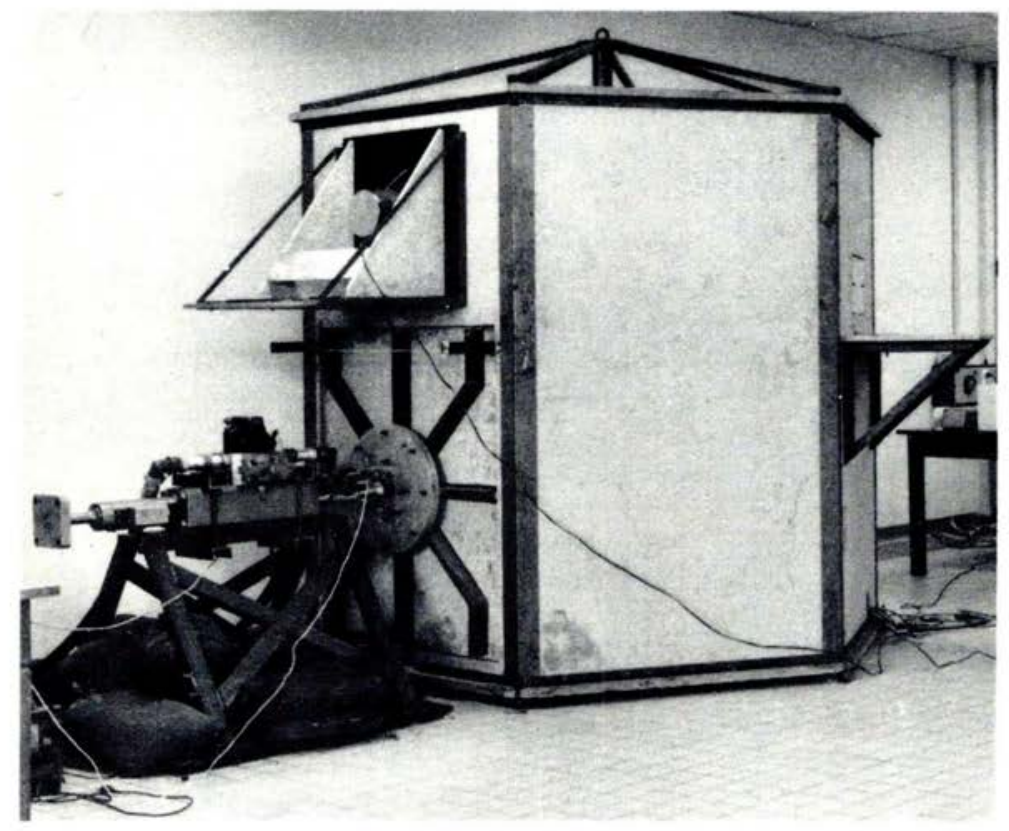

Fig. 2. - Caisson à infra-sons utilisé par le C.R.M.A.

spectrale des pressions explorée en tiers d'octave met en évidence la bonne émergence du signal infra-sonore fondamental par rapport au spectre harmonique d'accompagnement (fig. 3). Il n'empêche que le spectre sonore parasite lié à la distorsion harmonique risque de contribuer, pour l'essentiel, à l'impression subjective ressentie par le sujet. Une contre-épreuve s'impose donc et le haut-parleur sert à restituer dans l'enceinte une ambiance acoustique identique à la précédente mais débarrassée de sa fondamentale infra-sonore. L'absence d'un tel traitement placebo risque de fausser l'analyse des résultats comme nous l'avons plusieurs fois souligné $[2,3]$.

De tels dispositifs, comme la Langley low-frequency noise facility [9] ou la dynamic pressure chamber de l'Aerospace Medical Research Laboratory de Wright-Patterson AFB [29] réalisent parfaitement la simulation des variations de pression liées au phénomène infra-sonore. Il faut remarquer, cependant, que 
pression et vitesse de vibration des particules ne sont en phase qu'à une distance de la source égale à une longueur d'onde. De tels dispositifs ne simulent donc pas le flux d'énergie vibratoire de basse fréquence propagé en milieu aérien, tel qu'on peut le rencontrer dans la nature ou au niveau de certains postes de travail. Dans l'interprétation des résultats des expériences obtenues avec ces dispositifs, il est donc permis de se poser la question de savoir si, en fait, l'effet des infra-sons ne peut pas être, en partie, lié au flux d'énergie vibratoire rencontré et éventuellement absorbé par le sujet. Observons, cependant, que pour une fréquence de $10 \mathrm{~Hz}$, la longueur d'onde $\lambda=C / f$, est de l'ordre de $34 \mathrm{~m}$, ce qui, évidemment, pose des problèmes de réalisation pratique pour un dispositif expérimental susceptible d'accueillir un sujet humain. De plus, le mauvais rendement des émetteurs techniques d'infra-sons doit être pris en compte. De ce point de vue, le Pr GavreAu avait réalisé de bons simulateurs, mais ils étaient de diamètre modeste $[10,11]$.

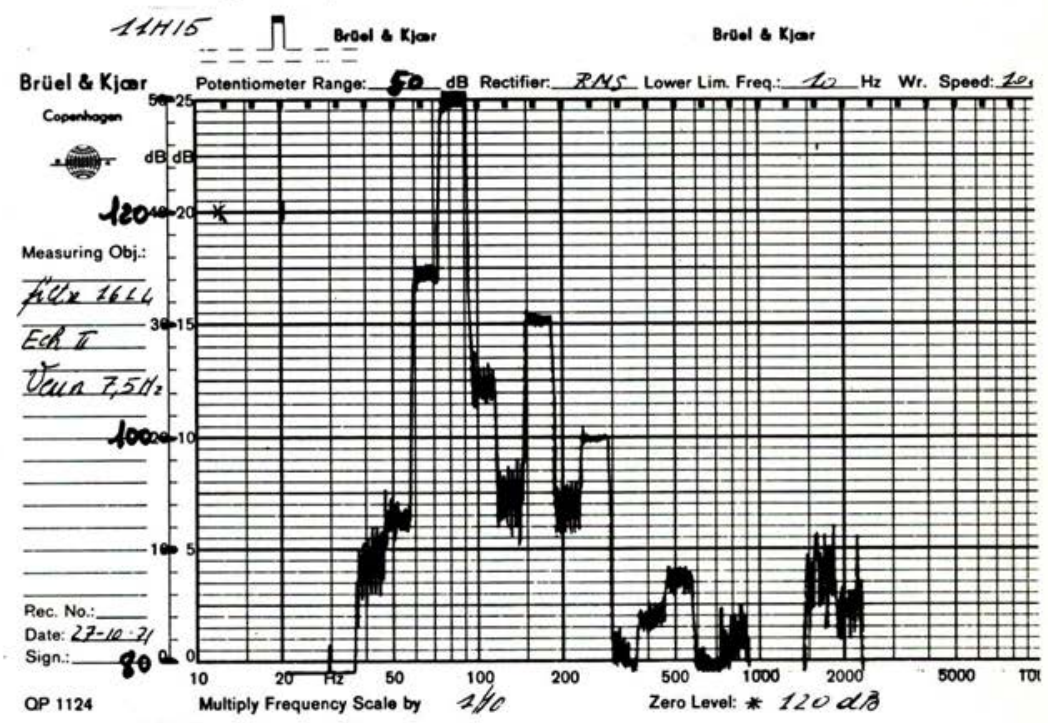

FIG. 3. - Spectre recueilli lors du fonctionnement du piston hydraulique (filtre B et K, type 1614 et amplificateur B et K, type 2606).

Une dernière remarque s'impose à propos de l'utilisation des dispositifs expérimentaux ou du recueil d'observations au niveau de certains postes de travail. Il est évident que les sources expérimentales ou industrielles d'infra-sons créent une excitation des solides qui les supportent : sol de l'atelier ou parois du caisson expérimental. Le sujet dont on observe les réactions risque d'être ainsi sollicité par des vibrations de fréquences infra-sonores qui lui parviennent les unes directement par voie solidienne (sol-pieds par exemple), les autres par voie aérienne. Les énergies mises en jeu dans ces deux modes de transmission ne sont pas, en règle, comparables. Le cas de la transmission solidienne est traité en général sous le titre tolérance aux vibrations. Nous ne considérerons, ici, que l'effet des vibrations mécaniques propagées dans l'air. 


\section{$2^{\circ}$ Les résultats}

Plutôt que de livrer un catalogue fastidieux de tous les effets qui ont pu être rapportés dans une littérature qui peut souvent être qualifiée, comme le soulignent HARRIS, SOMMER et JOHNSON [17], de littérature à sensation, nous résumerons essentiellement les travaux publiés dans le compte rendu du premier colloque international sur les infra-sons organisé à Paris en septembre 1973 par le C.N.R.S. et le G.A.L.F., en nous intéressant tout particulièrement aux publications émanant de l'Aerospace Medical Research Laboratory, WrightPatterson AFB. Sauf indication contraire, l'indication de la fréquence correspond, dans ce qui suit, à un signal infra-sonore sinusoïdal. La fréquence est donnée en hertz $(\mathrm{Hz})$. Le niveau est donné en décibels $(\mathrm{dB})$ : nombre de décibels $=20 \log \left(P_{i} / P_{0}\right), P_{i}$ est la pression efficace du signal considéré, $P_{0}$ est la référence internationale $2.10^{-5}$ pascal $(\mathrm{Pa})[25]$.

\section{a) Chez les animaux}

Dès 1958, Grognot et coll. [16], utilisant une chambre à compression construite par PIMONOw [28], s'intéressent aux conséquences respiratoires d'une exposition à des niveaux infra-sonores élevés. Chez des rats soumis pendant $7 \mathrm{~min}$ à des niveaux de $174 \mathrm{~dB}$ (7 Hz-sinus) un aspect congestif des poumons est observé; les animaux soumis pendant 15 min à un niveau de $180 \mathrm{~dB}$ ( $7 \mathrm{~Hz}$-sinus) présentent de franches suffusions hémorragiques intra-alvéolaires.

Sur le chien anesthésié et intubé, JoHnson note essentiellement, à partir du niveau $160 \mathrm{~dB}$ (réf. $2 \cdot 10^{-5} \mathrm{~Pa}$ ), un effet sur le rythme respiratoire [19]. A partir des niveaux 164-166 dB, la ventilation diminue et les mouvements respiratoires spontanés cessent pour un niveau de $172 \mathrm{~dB}$.

Pour des fréquences inférieures à $1 \mathrm{~Hz}$, il n'y a plus de mouvement de la cage thoracique. Le système thoraco-pulmonaire se comporte comme une bouteille rigide dont la ventilation est assurée par les variations de pression dans le caisson qui entraînent un écart d'environ $23 \mathrm{p}$. cent entre le maximum et le minimum de la densité de l'air dans l'enceinte. Ceci est suffisant pour déterminer un mouvement de va-et-vient des gaz dans le système broncho-pulmonaire qui entraîne un débit moyen comparable à celui requis pour assurer une ventilation convenable chez le chien. Ces considérations sont à rapprocher des observations de MoHr et coll. [22] sur des sujets humains exposés à des niveaux infra-sonores de $153 \mathrm{~dB}$ et qui ressentaient une sensation de mouvement d'air dans la gorge.

Au-dessus de $1 \mathrm{~Hz}$, la cage thoracique est à nouveau mobilisée passivement et, vers $3-4 \mathrm{~Hz}$, le mouvement atteint son maximum, ce qui correspond aux fréquences de résonance de la cage thoracique et du diaphragme, déterminées sur table vibrante, chez le chien anesthésié, par NiCKERSON et COERMAN en 1962 [23]. Il faut remarquer que, pour un signal sinusoïdal, le nombre de décibels de 172, correspond sensiblement à une pression efficace de $79,43 \cdot 10^{2} \mathrm{~Pa}$, soit une pression crête de $112,33.10^{2} \mathrm{~Pa}$.

Ces chiffres sont à rapprocher de ceux rencontrés en mécanique ventilatoire et du principe de respiration en équipression. On sait qu'un sujet humain immergé sous l'eau et respirant par l'intermédiaire d'un tuba ne pourra guère, 
par cet artifice, dépasser une profondeur de $1,50 \mathrm{~m}$. A cette profondeur, par rapport à la pression buccale $P_{b}$ qui reste égale à la pression $P_{B}$ qui règne à la surface, le thorax est soumis à une pression statique supplémentaire d'environ $150 \cdot 10^{2} \mathrm{~Pa}$. Dans ces conditions, l'inspiration est impossible. Les muscles inspiratoires sont incapables de lutter contre ce déséquilibre de pression et de recréer, entre pression buccale et pression alvéolaire, le modeste gradient de pression nécessaire à la mobilisation d'un volume courant.

Comme le souligne JoHnSON, ces considérations restent académiques, d'une part parce que de telles intensités infra-sonores ne se rencontrent pas actuellement en dehors de circonstances expérimentales particulières et, d'autre part, parce que le chien éveillé exposé à ces niveaux ne voit pas son rythme respiratoire modifié [19]. Des chiens, non anesthésiés, ont parfaitement toléré des expositions d'une durée de $5 \min$ à $172 \mathrm{~dB}(2,3,4,5,6$ et $8 \mathrm{~Hz})$, à $166 \mathrm{~dB}$ $(16 \mathrm{~Hz})$ et à $158 \mathrm{~dB}(32 \mathrm{~Hz})$. Un même chien a subi, pendant 14 heures réparties sur une période de 6 semaines, des expositions répétées d'une durée de 1 heure pour des fréquences de $1,2,4,8,16$ et $30 \mathrm{~Hz}$, sans qu'on puisse mettre en évidence, chez cet animal, la moindre manifestation pathologique à l'autopsie; malheureusement, l'oreille de cet animal n'a pas été examinée. Du point de vue cardio-vasculaire, chez le chien, JOHNSON ne fait aucune remarque particulière alors que LEMAIRE et coll. [21] observent des modifications tensionnelles chez le chien anesthésié soumis à des niveaux infra-sonores élevés à la fréquence de $5 \mathrm{~Hz}$.

Les effets sur le système auditif ont été étudiés chez le chinchilla exposé pendant $32 \mathrm{~min}$ à $2 \mathrm{~Hz}$, ou pendant $8 \mathrm{~min}$ à $8 \mathrm{~Hz}$ [19]. Pour des niveaux de $172 \mathrm{~dB}$, la rupture tympanique est observée de façon constante et les muscles de l'oreille moyenne présentent une suffusion hémorragique. Par contre, à $160 \mathrm{~dB}$, aucune lésion n'est observée. Au cours des expositions à $172 \mathrm{~dB}$ comme à $160 \mathrm{~dB}$, l'équilibre des animaux ne paraît absolument pas perturbé, même lorsqu'ils se dressent sur leurs pattes de derrière. PARKER et coll. enregistrent les pressions périlymphatiques, chez le cobaye et le singe [27]. Les modifications des pressions périlymphatiques reproduisent celles du stimulus infra-sonore pour les fréquences allant de 0,5 à $2 \mathrm{~Hz}$ et pour les audio-fréquences graves de 40 à $50 \mathrm{~Hz}$. Entre ces deux domaines de fréquences, la stimulation donne lieu à des variations complexes des pressions périlymphatiques. Quoi qu'il en soit, pour des stimulations aussi intenses que $172 \mathrm{~dB}$ à 1,2 et $4 \mathrm{~Hz}, 169 \mathrm{~dB}$ à $10 \mathrm{~Hz}$ et $162 \mathrm{~dB}$ à $20 \mathrm{~Hz}$, aucun mouvement oculaire induit par la stimulation vestibulaire ne peut être mis en évidence, chez le cobaye ou le singe, alors qu'une stimulation dans la gamme sonore induit une réponse pour des niveaux considérablement plus faibles. Ainsi, à des intensités de 140 à $145 \mathrm{~dB}$ et une fréquence de $500 \mathrm{~Hz}$, le singe présente un nystagmus net et reproductible.

En ce qui concerne le comportement des animaux exposés à de tels niveaux, JoHNSON souligne qu'il n'a vraiment rien d'extraordinaire, même dans le cas des chinchillas qui ont présenté une rupture tympanique bilatérale [19]. Certes, il a été rapporté récemment l'effet d'une stimulation infra-sonore sur le test de nage de la souris, mais on connaît les difficultés méthodologiques qui doivent être surmontées pour interpréter valablement une réduction observée du temps de nage chez la souris [8]. 
b) Chez l'homme

Chez l'homme, le seuil de douleur de l'oreille en pression statique est de 175 à $180 \mathrm{~dB}$; il est d'environ $150 \mathrm{~dB}$ à $10 \mathrm{~Hz}$ mais il faut remarquer avec NIXON [24] que pour des signaux infra-sonores, une légère sensation douloureuse n'implique pas l'existence d'un risque pour la fonction auditive.

NIXON utilisant un pistonphone analogue au dispositif stéthacoustique utilisé par GrogNOT et coll. [15] observe, chez un sujet sur trois, une baisse modérée et transitoire du seuil d'audition tonal aérien (T.T.S.-26), pour des expositions de $5 \mathrm{~min}$ à $135 \mathrm{~dB}, 18 \mathrm{~Hz}$ ou à $140 \mathrm{~dB}, 14 \mathrm{~Hz}$ [24]. La récupération est totale dans les 30 min suivant la fin de l'exposition. Ces deux stimulations comportaient des harmoniques dans la bande 20 à $100 \mathrm{~Hz}$ à un niveau tel qu'ils contribuaient certainement à l'apparition des T.T.S. observés. Reprenant ces expériences avec l'enceinte de l'Aerospace Medical Research Laboratory, NIXON, pour des expositions d'une durée inférieure à $8 \mathrm{~min}$ n'observe aucun T.T.S., même pour des niveaux aussi élevés que $150 \mathrm{~dB}$ à 1, 2, 4, 6, 8 et $15 \mathrm{~Hz}$. Quelques T.T.S. modérés et à récupération rapide sont observés pour des expositions supérieures à $20 \mathrm{~min}$ et des niveaux de 140 à $150 \mathrm{~dB}(4,7$ et $10 \mathrm{~Hz}$ ). Ces données concordent avec celles de MoHr et coll. [22] dont les expériences comportent des expositions brèves d'environ 2 min à des niveaux de 150-154 dB et avec celles de BORREDON et coll. [6] qui concernent des expositions d'une durée de 50 min à un niveau de $130 \mathrm{~dB}(7,5 \mathrm{~Hz})$. Dans ces deux conditions l'audiogramme, pris 1 heure après la fin de l'exposition, ne montre pas de T.T.S.

En se basant sur un nombre important d'expériences, NixON a pu proposer, en fonction de la durée d'exposition, des niveaux maximaux à ne pas dépasser pour éviter l'apparition de T.T.S. chez le sujet sain (tableau I). Bien entendu, ce type de recommandation ne s'applique qu'à des sujets indemnes de toute manifestation pathologique ou de toute séquelle d'intervention chirurgicale sur la cochlée.

TABLEAU I

Niveaux maximaux (en dB), Qu'il CONVIENT DE NE PAS dÉPASSER POUR PROTÉGER L'AUDITION, D'APRÈS NIXON [24]

\begin{tabular}{|c|c|c|c|c|c|c|c|c|}
\hline \multirow{2}{*}{$\begin{array}{c}\text { Durée } \\
\text { d'exposition } \\
\text { par } 24 \mathrm{~h}\end{array}$} & \multicolumn{7}{|c|}{ Fréquence $(\mathrm{Hz})$} \\
\cline { 2 - 9 } & 1 & 5 & 7 & 8 & 10 & 11 & 12 & 20 \\
\hline $8 \min \ldots \ldots \ldots$ & 150 & 150 & 150 & 145 & 145 & 145 & 140 & 140 \\
1. heure........... & 145 & 138 & - & - & 135 & - & - & 132 \\
8 heures....... & 136 & 129 & - & - & 126 & - & - & 123 \\
24 heures....... & 131 & 124 & - & - & 121 & - & - & 118 \\
\hline
\end{tabular}

L'effet vestibulaire d'une stimulation infra-sonore a été particulièrement étudié par l'équipe de vON GIERKE en testant l'équilibre de sujets humains sur des rails de largeur variable [13]. La stimulation est soit bilatérale, soit portée sur une seule oreille. $\mathrm{Si}$, avec une stimulation dans la gamme sonore, un effet peut être détecté dès le niveau $95 \mathrm{~dB}$ et devenir majeur pour des niveaux de $140 \mathrm{~dB}$, aucun effet n'a pu être mis en évidence pour des intensités aussi élevées que 142 à 
$155 \mathrm{~dB}$ pour les fréquences de $0,6,1,6,2,4$ et $7 \mathrm{~Hz}$ et des expositions de 2 à 5 min. Quant à JoHNSON [19] il souligne qu'il a personnellement subi une stimulation unilatérale de $168 \mathrm{~dB}$ à $7 \mathrm{~Hz}$ pendant 1 min en conservant l'équilibre sur un seul pied et sans apparition de nystagmus ni sensation d'oscillation. Il est assez remarquable d'observer que chez l'animal (cobaye, singe) comme chez l'homme, contrairement à ce que l'on pouvait imaginer, le vestibule présente un seuil considérablement plus élevé pour un stimulus infrasonore que pour une stimulation sonore.

En ce qui concerne les effets sur l'acuité visuelle, on sait qu'en fait, les fréquences de résonance des globes oculaires sont nettement supérieures aux fréquences infra-sonores et le problème se pose entre 40 et $70 \mathrm{~Hz}[13,27]$.

SLARVE et JoHNSON [29] ont rapporté récemment une très intéressante étude concernant l'exposition de 4 sujets humains à des niveaux infra-sonores élevés. Chaque séance a une durée de $8 \mathrm{~min}$, chaque sujet est exposé en moyenne une fois par semaine et au total il y a 55 expériences de 15 types différents selon le schéma suivant :

$\begin{array}{rrlll}30 \mathrm{~Hz}-125 \mathrm{~dB} & 10 \mathrm{~Hz}-144 \mathrm{~dB} & 8 \mathrm{~Hz}-144 \mathrm{~dB} \\ 25 \mathrm{~Hz}-132 \mathrm{~dB} & 8 \mathrm{~Hz}-120 \mathrm{~dB} & 6 \mathrm{~Hz}-144 \mathrm{~dB} \\ 20 \mathrm{~Hz}-135 \mathrm{~dB} & 8 \mathrm{~Hz}-126 \mathrm{~dB} & 4 \mathrm{~Hz}-144 \mathrm{~dB} \\ 16 \mathrm{~Hz}-139 \mathrm{~dB} & 8 \mathrm{~Hz}-132 \mathrm{~dB} & 2 \mathrm{~Hz}-144 \mathrm{~dB} \\ 12,5 \mathrm{~Hz}-142 \mathrm{~dB} & 8 \mathrm{~Hz}-138 \mathrm{~dB} & 1 \mathrm{~Hz}-144 \mathrm{~dB}\end{array}$

Deux effets sont observés de manière constante :

- modulation de la voix quand le sujet parle dans un microphone;

- sensation d'augmentation de pression dans l'oreille moyenne cédant temporairement à la manœuvre de Valsalva.

Il y a 34 observations de vibrations de la poitrine et de la paroi abdominale pour des fréquences comprises entre 4 et $25 \mathrm{~Hz}$ et, à $8 \mathrm{~Hz}$, elles apparaissent dès le niveau $132 \mathrm{~dB}$.

BORREDON et coll. $[2,4]$ étudient particulièrement les paramètres cardiovasculaires de 42 jeunes hommes exposés pendant $50 \mathrm{~min}$ à $130 \mathrm{~dB}$ 7,5 Hz. Subjectivement, les 43 sujets tolèrent parfaitement cette exposition. Une augmentation légère, mais significative, de la pression artérielle diastolique est néanmoins mise en évidence. On ne peut affirmer que la fondamentale infrasonore ait joué un rôle exclusif dans l'apparition de cette augmentation de la pression artérielle. Cette observation n'en vient pas moins sérieusement étayer la mauvaise réputation du travail en atmosphère bruyante pour ce qui concerne l'incidence de l'apparition de l'hypertension artérielle dans une population de travailleurs.

En ce qui concerne le comportement, une légère tendance à la somnolence est remarquée. Elle est entretenue par une sensation auditive de "pulsation sonore ", à la fréquence fondamentale, analogue à celle qui peut être ressentie dans la chambre des machines diesels d'un navire. Cependant, le temps de réponse à une sollicitation lumineuse qui est l'indice comportemental étudié n'est pas perturbé [2]. Harris et coll., dans une publication récente [17], font 
justice de publications rapportant, chez l'homme, des effets comportementaux pour le moins étonnants et imputés à des expositions à des niveaux infra-sonores modérés. Aussi nous rallierons-nous aux conclusions de HARRIS et coll. qui considèrent que les normes édictées à l'issue du colloque international, tenu à Paris en septembre 1973 sous l'égide du C.N.R.S. et du G.A.L.F., restent valables ( fig. 4) [17]. Sur cette figure, à titre de repère par rapport aux normes de protection reconnues dans le domaine des bruits audibles, on a indiqué l'origine de la courbe NR 85 d'évaluation du bruit (NF S 30-010). Dans les courbes NR, les niveaux de pression acoustique sont mesurés par bande d'octave. Ces courbes ne sont normalisées que pour les bandes d'octaves de fréquence centrale 31,5 , $63,125 \mathrm{~Hz}$, etc. En deçà de la fréquence 31,5 , la courbe NR 85 a donc été extrapolée jusqu'à la limite de séparation des effets significatifs des infra-sons, limite qui s'était dégagée à l'issu du colloque.

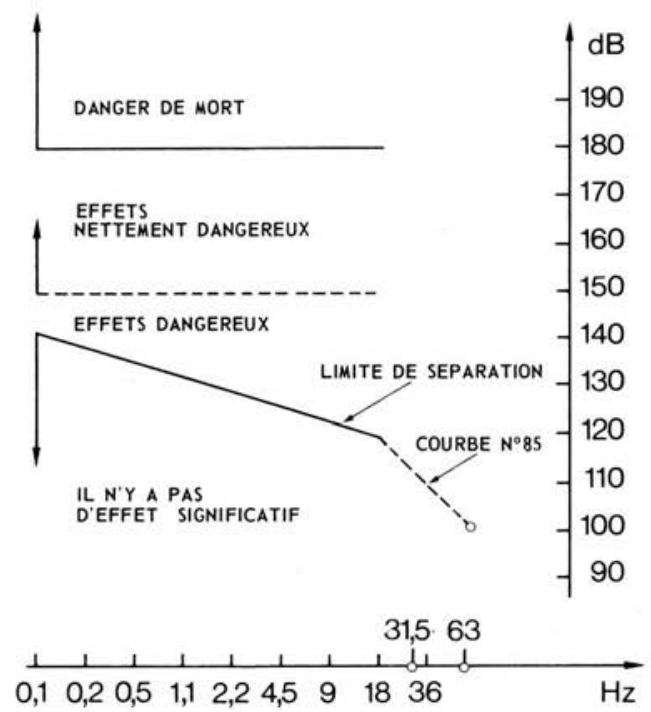

FIG. 4. - Niveaux infra-sonores et limites des effets à prendre en considération (d'après STAN A., Colloque international $n^{\circ} 232$ sur les infra-sons, Paris, éditions du C.N.R.S., 1974).

\section{IV. - CONCLUSIONS}

En conclusion à cet article destiné à une revue consacrée à la protection, il nous reste à dire un mot des problèmes posés par celle-ci. Dans le domaine sonore et du point de vue énergétique c'est-à-dire en dehors de toute considération sur la gêne, la protection individuelle est relativement aisée puisqu'elle concerne exclusivement l'appareil sensoriel cochléaire. Il existe actuellement sur le marché des moyens de protection individuels présentant un bon compromis entre efficacité, confort et prix. Cependant, ces moyens de protection ont une efficacité modérée vis-à-vis des fréquences graves. Dans le domaine infra-sonore, la seule loi d'affaiblissement qui reste à prendre en 
considération est la loi de masse, ce qui implique un poids rédhibitoire pour un moyen de protection individuel. De plus, la cochlée n'a peut-être plus, vis-à-vis de l'agression, le seuil de réponse et/ou le seuil lésionnel le plus bas. Par conséquent, si une protection individuelle associant bouchon d'oreille et coque auriculaire peut encore être préconisée [24], il n'en demeure pas moins que dans ce domaine, seuls doivent être pris en considération la réduction à la source ou les moyens de protection collectifs. C'est dire la nécessité d'une étude ergonomique des postes de travail dès le stade de leur conception par l'ingénieur. La correction a posteriori, par le port de moyens de protection individuels, d'une erreur de conception du poste de travail, ne peut être implicitement admise comme il advient trop souvent, malheureusement, dans le cas de postes de travail soumis à une nuisance sonore.

\section{BIBLIOGRAPHIE}

[1] Bonnardel G. Les infra-sons et les transports. Rapport de l'Institut de Recherche des Transports, Bron, 1977, 79 p.

[2] BorRedon P., NATHIE J. Réactions physiologiques de sujets humains exposés à des infrasons. Rapport $\mathrm{n}^{\circ}$ 3713/EASSAA/CRMA/RECH, 8 décembre 1972, 58 p.

[3] Borredon P., Nathie J. Effets physiologiques observés chez l'homme exposé à des niveaux infra-sonores de $130 \mathrm{~dB}$. Dans : Colloque international $n^{\circ} 232$ sur les infra-sons, Paris, septembre 1973. Paris, Éditions du C.N.R.S., 1974, 61-84.

[4] Borredon P., Nathie J., Gibert A. Étude, chez l'homme, des effets physiologiques d'une exposition à des niveaux infra-sonores de $130 \mathrm{~dB}$. Dans : Vibration and combined stresses in advanced systems (Von Gierke H. E., Ed.). Rapport AGARD-CP n 145. Neuilly, OTAN, 1975, B 28, 1-13.

[5] Borredon P., Santucci G. Le problème du bruit dans les Armées. Médecine et Armées, 1975, 3 (9), 733-738.

[6] Borredon P., Nathie J. Audiogramme et exposition à des variations infra-sonores de la pression aérienne. Rev. Méd. Aéron. spat., 1975, 13 (53), 13-19.

[7] Bryan M., Tempest W. Does infra-sound make drivers « drunk "? New scientist, 1972, 53, 584-586.

[8] Busnel R. G., Lehmann A. Séparation des effets psychophysiologiques des infra-sons par l'utilisation d'animaux génétiquement sourds. Communication au G.A.L.F., Paris, 25 novembre 1976.

[9] Edge P. M., Jr., Mayes W. H. Description of Langley low-frequency noise facility and study of human response to noise frequencies below $50 \mathrm{cps}$. NASA Technical Note D. 3204, 1966.

[10] Gavreau V. Sons graves intenses et infra-sons. Effets physiologiques, protection. Science et Progrès, La Nature, 1968, $\mathrm{n}^{\circ} 3401,336-344$.

[11] Gavreau V., Condat R., Saul H. Infra-sons : générateurs, détecteurs, propriétés physiques, effets biologiques. Acustica, 1966, 17, 1-10.

[12] Von Gierke H. E. Dynamic characteristics of the human body. Dans : Perspectives in biomedical engineering (Kenedi R. M., Ed.). Londres, MacMillan, 1973, 193-202.

[13] Von Gierke H. E. Effects of infrasound on man. Dans : Colloque international $\mathrm{n}^{\circ} 232$ sur les infra-sons, Paris, septembre 1973. Paris, Éditions du C.N.R.S., 1974, 419-433.

[14] Grandjean E. Précis d'ergonomie. Organisation physiologique du travail. Paris, Dunod, 1969. 
[15] Grognot P., Nathie J., Borredon P., Chevaleraud J. Réactions de certaines fonctions visuelles de l'homme soumis à l'action de vibrations infra-sonores portées uniquement au niveau du tympan. Rapport $\mathrm{n}^{\circ}$ 1700/CERMA/RECH., 1969.

[16] Grognot P., Senelar R., Loubiere R. Action des vibrations infra-sonores sur le poumon de rat. Rapport C.R.M.A. ${ }^{\circ}$ 1057, 1958.

[17] Harris C. S., Sommer H. C., Johnson D. L. Review of the effects of infrasound on man. Aviat. Space Environ. Méd., 1976, 47, 430-434.

[18] Humbert D. Les infra-sons mortels des Bermudes. Le Point, 1976, n 222, p. 68.

[19] Johnson D. L. Various aspects of infrasound. Dans : Colloque international $n^{\circ} 232 \mathrm{sur}$ les infra-sons, Paris, septembre 1973. Paris, Éditions du C.N.R.S., 1974, 337-355.

[20] Kravtchenko A. On suspecte les infra-sons. Revue Militaire soviétique, 1975, $\mathrm{n}^{\circ} 10$, 28-29.

[21] Lemaire R., Grognot P., Fabre J. Étude expérimentale de l'hypotension artérielle consécutive à l'exposition aux vibrations aériennes infra-sonores. C. R. Soc. Biol. Paris, $1965,159,629-630$.

[22] Mohr G. C., Cole J. N., Guild E., von Gierke H. E. Effects of low frequency and infrasonic noise on man. Aerospace Med., 1965, 36, 817-824.

[23] Nickerson J. L., Coermann R. R. Internal body movements resulting from externally applied sinusoidal forces. Rapport $\mathrm{n}^{\circ}$ AMRL/TDR/62-81, Wright-Patterson AFB, Ohio, 1962.

[24] Nixon C. W. Human auditory response to intense infra-sound. Dans : Colloque international $n^{\circ} 232$ sur les infra-sons, Paris, Septembre 1973, Paris, Éditions du C.N.R.S., $1974,317-335$.

[25] AFNOR. Vocabulaire de l'acoustique, définitions générales. Norme française homologuée, NF S 30-101, 1973.

[26] AFNOR. Vocabulaire de l'acoustique, acoustique physiologique et psycho-acoustique. Norme française homologuée NF S 30-105, 1975.

[27] Parker D. E., Reschke M. F., TubBs R. L. Effects of sound on the vestibular system. Dans : The use of nystagmography in aviation medicine (Guedry F. E., Ed.). Rapport AGARD-CP $\mathrm{n}^{\circ} 128$. Neuilly, OTAN, 1973, A 16, 1-12.

[28] Pimonow L. Les infra-sons. Paris, Éditions du C.N.R.S., 1976.

[29] Slarve R. N., Johnson D. L. Human whole-body exposure to infra-sound. Aviat. Space Environ. Med., 1975, 46, 428-431.

[30] DE LA TAILle R. La pollution par les infra-sons. Science et Vie, 1977, 129, 20-25.

[31] Westin J. B. Infrasound: a short review of effects on man. Aviat. Space Environ Med., 1975, 46, 1135-1143. 